\title{
Potential Benefits of Increased Public Transit Ridership in Medium Sized Cities: A Case Study
}

\author{
Taniya Sultana1, Virginia P. Sisiopiku², Jalal Khalil ${ }^{3}$, Da Yan ${ }^{3}$ \\ ${ }^{1}$ Department of Civil \& Environmental Engineering, Louisiana State University, Baton Rouge, LA, USA \\ ${ }^{2}$ Department of Civil, Construction and Environmental Engineering, University of Alabama at Birmingham, Birmingham, \\ AL, USA \\ ${ }^{3}$ Department of Computer Science, University of Alabama at Birmingham, Birmingham, AL, USA \\ Email: vsisiopi@uab.edu
}

How to cite this paper: Sultana, T., Sisiopiku, V.P., Khalil, J. and Yan, D. (2022) Potential Benefits of Increased Public Transit Ridership in Medium Sized Cities: A Case Study. Journal of Transportation Technologies, 12, 59-79.

https://doi.org/10.4236/jtts.2022.121004

Received: December 1, 2021

Accepted: January 4, 2022

Published: January 7, 2022

Copyright $\odot 2022$ by author(s) and Scientific Research Publishing Inc. This work is licensed under the Creative Commons Attribution International License (CC BY 4.0).

http://creativecommons.org/licenses/by/4.0/

\begin{abstract}
At the beginning of the twentieth century, the United States was leading in the public transit sector, but following World War II, private automobiles became more affordable and gained popularity. Transportation infrastructure investments that increased road capacity further facilitated the increase in automobile use at the expense of reduced public transit ridership. With the increase of dependency on automobiles and the continuing growth of private automobile ownership and use, various problems became major challenges in big cities of USA. These include traffic congestion, air pollution, road and parking infrastructure costs, energy consumption, traffic safety, fewer mobility options for the non-drivers, and a decline in the image and use of public transit. This study uses a medium sized city, Birmingham as a case study to investigate the potential of public transit to reduce automobile trips and in turn improve the overall performance of the road network by addressing the abovementioned challenges. An agent-based simulation model was developed for the Birmingham metropolitan region using the Multi-agent Transport Simulation (MATSim) platform. Three scenarios were considered with gradually increased transit ridership to identify the benefits of increased public transit. Traffic volume, network average speed, and travel times were used as performance measures for the evaluation of the designated scenarios. Results suggest that modal shifts toward public transit and reduction in travel demand for an automobile can result in improvements in speed and travel time for all users. Therefore, investments for improving transit quality and frequency of service, as well as campaigns to improve the image of public transit and make it a mode of choice for transportation users can increase transit ri-
\end{abstract}


dership and, in turn, improve network operations, thus are deemed worthy for medium sized cities.

\section{Keywords}

Public Transit, Ridership, Modal Shift, Transportation Network Operations, MATSim

\section{Introduction}

Public transit is also known as public transport, urban transit, mass transit and public transportation. It includes a variety of transportation modes and services such as buses, trains, ferries, vanpools, paratransit, etc. which are available to general public [1]. Due to its higher occupancy, public transit moves travelers more efficiently than the automobile and can play a crucial role in addressing environmental and urban problems such as traffic congestion and greenhouse gas emissions. For example, 14 percent of global $\mathrm{CO}_{2}$ emissions by 2010 were solely attributed to the transportation sector [2] causing 2200 premature deaths and more than $\$ 18$ billion expenditures in public health in the US [3]. In 2019, greenhouse gas emissions from transportation accounted for about 29 percent of total U.S. greenhouse gas emissions, making it the largest contributor of U.S. greenhouse gas emissions

Even though the population of the United States nearly doubled from 1957 to 2017 (172 to 326 million), the number of transit trips over these 60 years remained almost unchanged (10.4 billion to 10.1 billion). The steady increase of private automobile use in the US, at the expense of transit, is attributed to many factors including the development of the US interstate system and the continued expansion of the transportation network infrastructure. Besides the comfort and flexibility of using automobiles, another important reason behind the reduction of transit ridership is urban sprawl. When affordable housing is far away from the job location and is spread in less densely populated areas, transit accessibility becomes limited thus leading to increased automobile use [4].

Many locations are rethinking the current transportation and urban planning model and considering the potential benefits from more dense development served by expanded transit options. Using Birmingham, Alabama as a case study, the objective of this study is to demonstrate potential benefits of shifting automobile trips by increasing public transit ridership. Despite an estimated Birmingham Metro population of over 1.1 M, the public transit options are currently limited to a bus transit system that has faced systemic problems of low ridership and lack of resources and revenues. Reasons behind these issues include the unfavorable image of transit use, lack of resources and support for public transit, and limited-service availability. Therefore, evaluating the potential benefits of increased transit ridership on transportation network operations might help to infer the worthwhile value of an investment for public transit in 
medium-sized cities like Birmingham.

\section{Literature Review}

Many studies examined and documented transportation users' travel preferences and practices. The main stated reasons for selecting the automobile as the preferred mode of transportation are privacy and flexibility, sense of independence, power, control, enjoyment and prestige [5]. Moreover, an automobile journey is fully under the control of the driver, who can drive alone or with chosen persons rather than unknown individuals [6]. These perceptions along with increased affordability of private automobiles in the US resulted in a continuous increase in the number of automobile trips and contributed to increased automobile ownership and automobile dependency [7]. Car ownership creates a strong commitment to use car as well as an attitude to undervalue the alternative transport modes and keep them away from more environmentally friendly public transportation [8].

This study explored underlying benefits of using public transportation as they pertain to individual transportation users, transportation authorities and the society. Some of them are summarized and documented in this paper.

\subsection{Environmental Benefits}

Public transportation can support sustainability initiatives by reducing the frequency of use of private cars and associated environmental impacts. As public transit transports people collectively it is found to produce $45 \%$ less $\mathrm{CO}_{2}, 95 \%$ less $\mathrm{CO}$, and $48 \%$ less $\mathrm{NO}_{2}$ than private vehicles [9]. A study after a rail system opening in Taiwan indicates that CO was reduced by $5 \%-15 \%$ and another environmental assessment after rail service expansion in Germany indicates the reduction of pollutants such as $\mathrm{NO}, \mathrm{NO}_{2}$ and $\mathrm{CO}$ [10]. It is estimated that if $5 \%$ of Americans used public transit instead of private car or if every American used public transit for 5\% of their trips during 1970 to 1998 , the CO pollution reduction would be more than all the $\mathrm{CO}$ emitted from all metal processing plants and chemical manufacturing section combined [11].

\subsection{Economic and Social Benefits}

According to a report published by American Public Transportation Association (APTA) in 2009, for one billion dollars of annual investment in public transportation, there would be more than $\$ 1.7$ billion dollars of added annual GDP [12]. Depending on some factors such as mileage reduction, declining vehicle ownership, etc., a shift from automobile to transit provides a variety of cost savings [13] including fuel and oil, insurance costs, parking costs, etc.

Available public transit services can be especially beneficial for people with low income who cannot afford automobile ownership and for elderly and disabled persons by offering convenient and affordable service. Thus, it increases social and economic opportunities for physically, socially and economically dis- 
advantaged people along with achieving equity objectives [1].

\subsection{Health Benefits}

According to the US Center for Disease Control (CDC) and prevention, at least 30 minutes of daily physical activity such as bicycling, or walking is necessary to stay healthy [14]. An Atlanta, Georgia survey results show that almost two-thirds of the recommended daily physical activity is achieved by the transit users which is ten times greater than the average walking reported by the non-transit users [15]. It is also worth noting that medical expenses are 32\% lower ( $\$ 1019$ per year) for adults who achieve the recommended physical activity than those who do not (\$1349 per year) [16]. According to another study, 21 minutes of walking can help to burn 65.1 to 98.7 calories and 100 kilocalories burn per day might save $\$ 12,500$ dollars per person in obesity-associated medical costs [17]. These findings clearly show the value of transit in the wellbeing of transportation users, an issue that is often overlooked by decision makers when they appropriate funding for transportation services and projects.

\subsection{Congestion Reduction}

The results from a seemingly unrelated regression equations (SURE) model showed that 50 percent increase of city bus routes in highly populated areas of Taiwan reduce car usage by $1.4 \%$ which corresponds to 300,000 vehicles [18]. Another study on Bay Area Rapid Transit (BART) system indicates that in absence of BART services during the morning peak, driving times increased more than four times in multiple corridors [19]. Similar studies [20] [21] on investigating the effects of transit absence indicated increased traffic volumes, and longer delays. On the contrary, a study on Salt Lake City's University TRAX light-rail system in 2014 found that typical vehicle traffic has reduced by nearly $50 \%$ with the expansion of the light rail system [22]. Some other studies [23] [24] also provide links between transit availability and transportation mode choices and highlight the potential positive impacts from introduction or expansion of transit services in a region, for individuals, the transportation network operations, and the community. However, given local differences, it is important to conduct local studies in order to gain an understanding of potential impacts of transit ridership increase on local congestion and quantify such impacts.

\section{Methodology}

\subsection{Simulation Platform}

Based our earlier work on comparisons of available simulation platforms [25] [26], the simulation platform selected for this study was the Multi-Agent Transport Simulation platform (MATSim). The platform is designed to simulate large-scale scenarios by adopting a computationally efficient queue-based approach [27]. It incorporates time choice, mode choice, and/or destination choice into an iterative loop, leading to a stochastic user equilibrium. The MATSim 
model is publicly hosted and allows for simulation of the impact of changes in transit ridership in medium sized cities such as Birmingham, AL.

An open-source software, MATSim requires its input files to be as XML files. Minimum input files required to run the software are: 1) Configuration file; 2) Network file; 3) Population/plans file. The configuration file builds the connection between MATSim tool and all other Extensible Markup Language (XML) files (e.g. network, population, etc.), and contains a list of settings that influence how the simulation behaves. MATSim's network file consists of nodes and links and describes the infrastructure that agents can use to move around. Nodes are defined by coordinates while the link requires definition of several attributes including the length of the link, capacity, speed, and the number of available lanes. The population file provides information about travel demand, e.g. a list of agents and their travel diaries. The travel demand is described by the daily plans of each agent. The population file contains a list of transportation users and their daily plans, activities, and legs.

Each simulation job executes in iterations where each iteration executes the selected plans of all agents over an underlying road network. It starts with an initial population demand (a.k.a. plans) in the studied area which is application-dependent. Each agent in the population maintains a pool of up to 5 day-plans. In each iteration, 1) MATSim's “mobsim” simulation executor runs the selected plans of the agents in the synthetic road network environment; 2) Then, a scoring function assigns a score to each plan based on the corresponding agent's experiences with the selected day plans (e.g., if congestion happens or not); 3) Afterwards, the replanning step selects a candidate plan based on the plan scores in each agent's day-plan pool, and may modify this plan for execution in the next iteration.

As far as simulation outputs are concerned, MATSim creates output data that can be used to monitor the current simulation setup progress as well as to analyze results. In each iteration, a linkstats file containing hourly count values and travel times on every network link is generated by the model. The user can specify the output interval of simulation statistics for individual links. MATSim provides overview summaries of counts and other statistics for the whole network, but also analyzes for individual links. Also, a google maps-based visualization is available, showing simulation output results for each station in a pop-up window. Details about MATSim are available in [27] and at https://www.matsim.org/.

\subsection{Case Study Design}

MATSim was used to develop a model considering private cars only (base) and three scenarios including public transit. The Population was generated by population synthesis [24] of travel diary from a survey and using open source data [28]. To speed up computational performance, and similarly to MATSIM-based studies reported in the literature [29] [30], 10\% of the total population was used for the simulation. Criteria considered for the development of each scenario are listed in Table 1. 
Table 1. Scenario design criteria.

\begin{tabular}{cccc}
\hline & Scenario 1 & Scenario 2 & Scenario 3 \\
\hline Probability of Choosing Public Transit (PT) & $\leq 0.1$ & $\leq 0.5$ & $\leq 0.9$ \\
Car Percentage & $97.5 \%$ & $87 \%$ & $76.4 \%$ \\
Public Transit Percentage & $1.1 \%$ & $5.7 \%$ & $10.1 \%$ \\
Walk Percentage & $1.4 \%$ & $7.3 \%$ & $13.5 \%$ \\
\hline
\end{tabular}

For the first scenario, the probability of choosing public transit is set as equal or less than 0.1 because with this probability, $1.1 \%$ of total agents (travelers) choose public transit. This percentage represents public transit ridership in Birmingham at the time of study [31] [32]. To determine the impact of increased ridership, the probability is then set as less than or equal to 0.5 and 0.9 respectively for scenario 2 and scenario 3, respectively. Table 1 also shows the expected market share of the various modes considered in the study (namely car, transit, and walk) under the 3 scenarios tested (namely probability of $\leq 0.1, \leq 0.5, \leq 0.9$ ).

\subsection{Model Calibration and Validation}

Simulation model calibration and validation are important steps toward increasing the confidence in use of any simulation model. Comparing traffic counts retrieved from the simulation model with actual traffic counts is an approach commonly used to validate simulation models, including MATSim [29]. In this study, traffic counts from the MATSim model (scenario 1) were compared to traffic volumes obtained from Alabama traffic data collected by the Alabama Department of Transportation (ALDOT) for two time periods (7 AM to 8 $\mathrm{AM}$ and $5 \mathrm{PM}$ to $6 \mathrm{PM}$ ). Traffic volume data used for this calibration were for selected freeway and state highway sections/links located in the Jefferson and Shelby counties. A total of 90 links along I-459 N, I-459 S, AL 25 S, I-65 N, AL 3 N, AL3 S, AL 5 E, AL 38 E, and the junction of I-65 and I-59 were considered for this validation.

Figure 1 and Figure 2 show model validation results, where the $\mathrm{X}$-axis represents the field volume and $\mathrm{Y}$-axis represents the simulated volumes. The three diagonal lines in the graphs represent the simulated versus real volume ratio of 2:1, 1:1 and 1:2 which are named as 2 counts, 1 count and 0.5 count respectively. Counts falling between 2 and 0.5 count are considered acceptable [33] and most of the data points are within these boundaries, thus the model validation is deemed acceptable.

\subsection{Link ID Selection}

MATSim generates output according to the link ID which refers to the identity of the roadway sections in the MATSim platform. This study analyzed the performance of road network as a result of increased transit ridership by examining links near the bus stops. The procedure used for selecting 93 links from the 1761 bus stop links within the study network is shown in Figure 3. 
Based on the data available at the live traffic website [34], traffic congestion in the Birmingham region peaks from 5 to $6 \mathrm{PM}$ for a typical weekday. During this time slot, an addition $50 \%$ of travel time is needed on average to complete a trip, compared to the travel time under free flow conditions. As far as congestion severity time period is concerned, the $5 \mathrm{PM}$ to $6 \mathrm{PM}$ period is followed by $4 \mathrm{PM}$ to $5 \mathrm{PM}, 3 \mathrm{PM}$ to $4 \mathrm{PM}, 7 \mathrm{AM}$ to $8 \mathrm{AM}$ and $8 \mathrm{AM}$ to $9 \mathrm{AM}$, during which travel times are $43 \%, 36 \%, 33 \%$ and $27 \%$ higher respectively, as compared to travel time under free flow conditions. The study selected the same five time periods for further analysis.

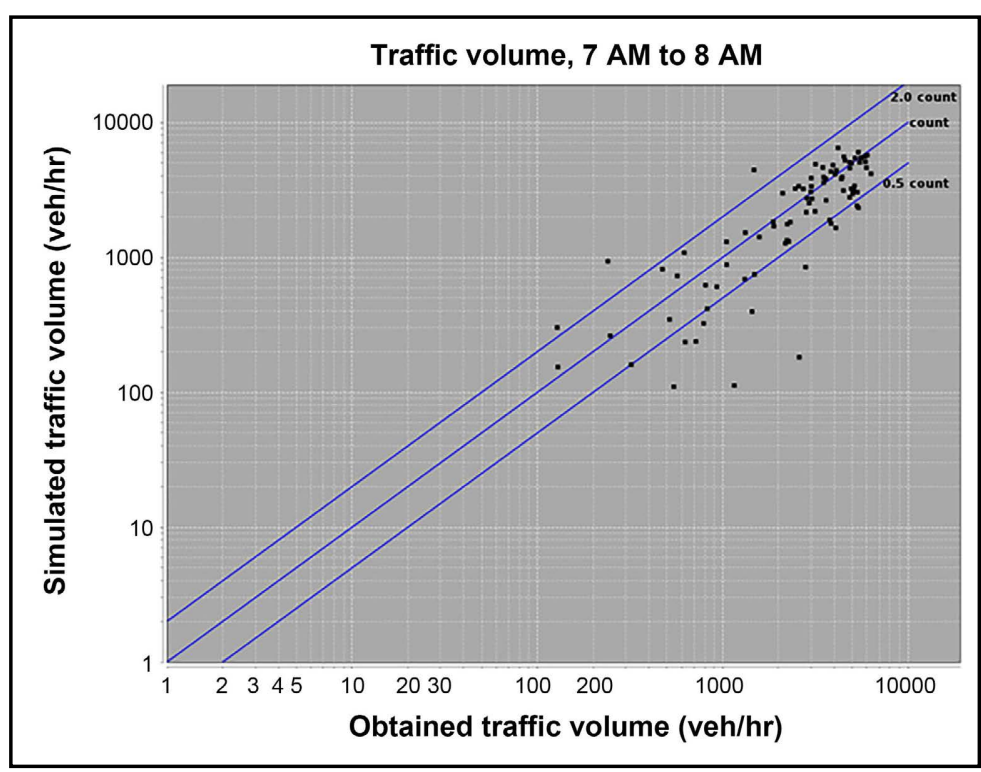

Figure 1. Comparison between simulated and obtained traffic volumes of validation links for $7 \mathrm{AM}$ to $8 \mathrm{AM}$.

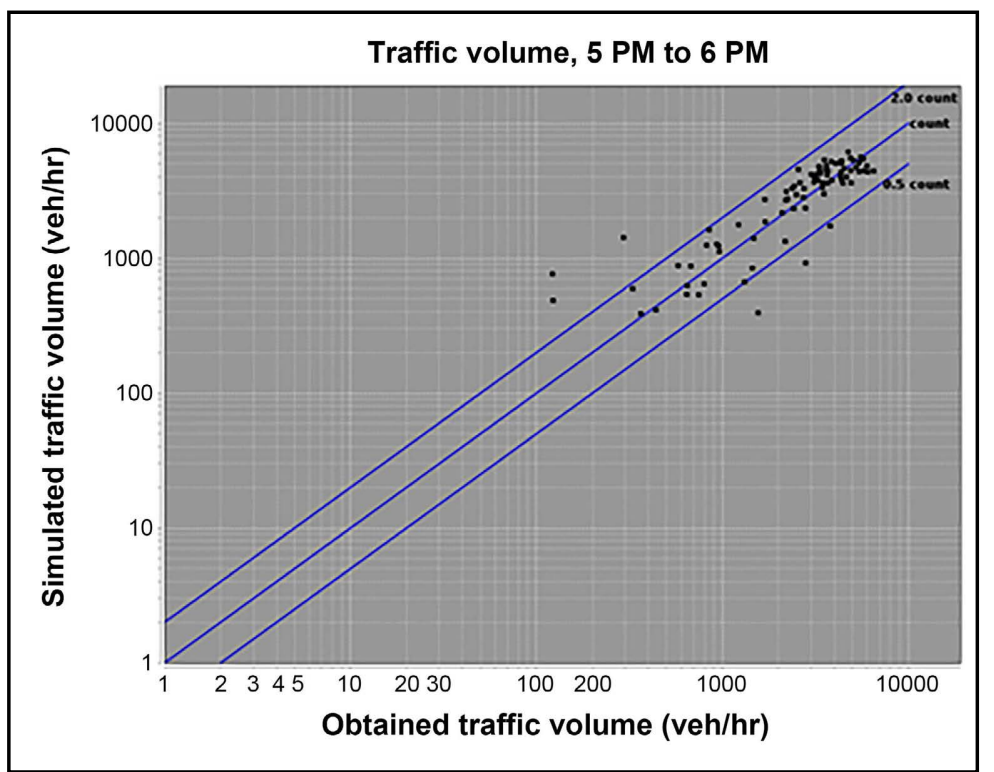

Figure 2. Comparison between simulated and obtained traffic volumes of validation links for $5 \mathrm{PM}$ to $6 \mathrm{PM}$. 


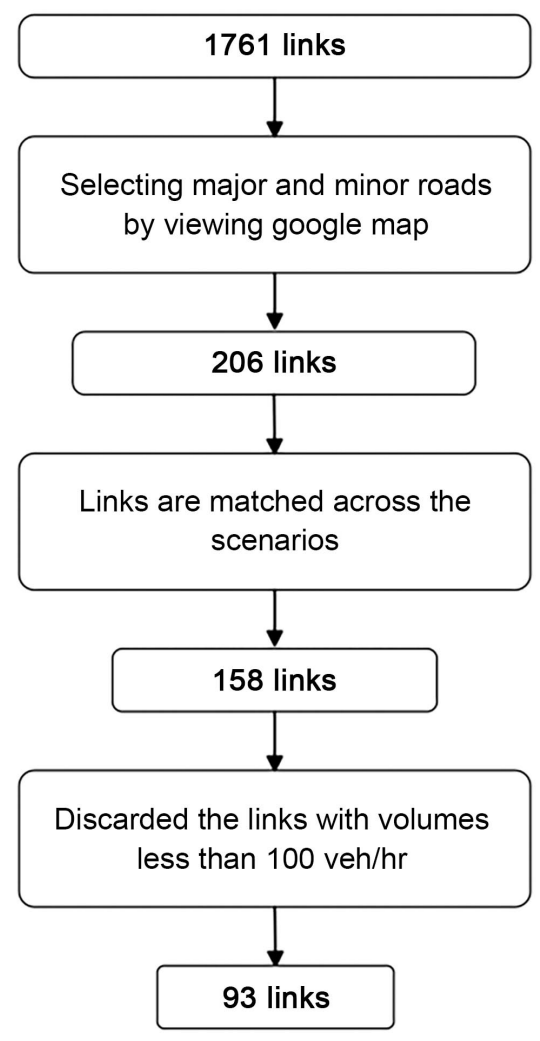

Figure 3. Flow chart of the link selection.

To accommodate the big range of volumes (120 veh/hr to $2520 \mathrm{veh} / \mathrm{hr}$ ), links were classified in five different groups based on the procedure described in Figure 4. Resulting link groupings from this procedure are summarized in Table 2.

It should be noted that the simulated traffic volumes are $10 \%$ of the total. For example, group 3 includes simulated volumes of $51-90 \mathrm{veh} / \mathrm{hr}$ which refer to links with actual traffic volumes of $510-900 \mathrm{veh} / \mathrm{hr}$ as seen in Table 2. Also, the volumes are total volumes per link (directional volumes, all lanes combined).

To determine the performance of the road network in the event of increased transit ridership, traffic volume, speed, and average travel time were analyzed for the selected 5 time slots. The results are summarized next.

\section{Results and Discussion}

\subsection{Traffic Volume Analysis}

Figure 5 shows the mean traffic volume for three of the scenarios, 5 time slots and 5 groups. The purpose of showing the traffic volume data for the study scenarios based on the groups is to allow for understanding how increased transit ridership affects network operations under different volumes levels. The black line, blue line, red line, green line and purple line in Figure 5 stands for the traffic volumes between $7 \mathrm{AM}$ to $8 \mathrm{AM}, 8 \mathrm{AM}$ to $9 \mathrm{AM}, 3 \mathrm{PM}$ to $4 \mathrm{PM}, 4 \mathrm{PM}$ to 5 $\mathrm{PM}$ and $5 \mathrm{PM}$ to $6 \mathrm{PM}$ respectively. To facilitate comparisons, the line color scheme used in the analysis represents the same time slots for all the groups. 


\subsubsection{Traffic Volume Change for Group 1}

Mean traffic volumes are shown for group 1 which consists of the study links with volumes of $101-300 \mathrm{veh} / \mathrm{hr}$. The probability of travelers choosing public transit increases from scenario 1 (public transit share of trips $=1.1 \%$ ) to scenario 2 (public transit share of trips $=5.7 \%$ ) and from scenario 2 to scenario 3 (public

Table 2. Grouping of the study links.

\begin{tabular}{ccc}
\hline Groups & Traffic Volume (veh/hr) & Number of Links \\
\hline Group 1 & $101-300$ & 22 \\
Group 2 & $301-500$ & 18 \\
Group 3 & $501-900$ & 20 \\
Group 4 & $901-1200$ & 17 \\
Group 5 & $>1200$ & 16
\end{tabular}

93 links with volume from 120 $\mathrm{veh} / \mathrm{hr}$ to $2520 \mathrm{veh} / \mathrm{hr}$

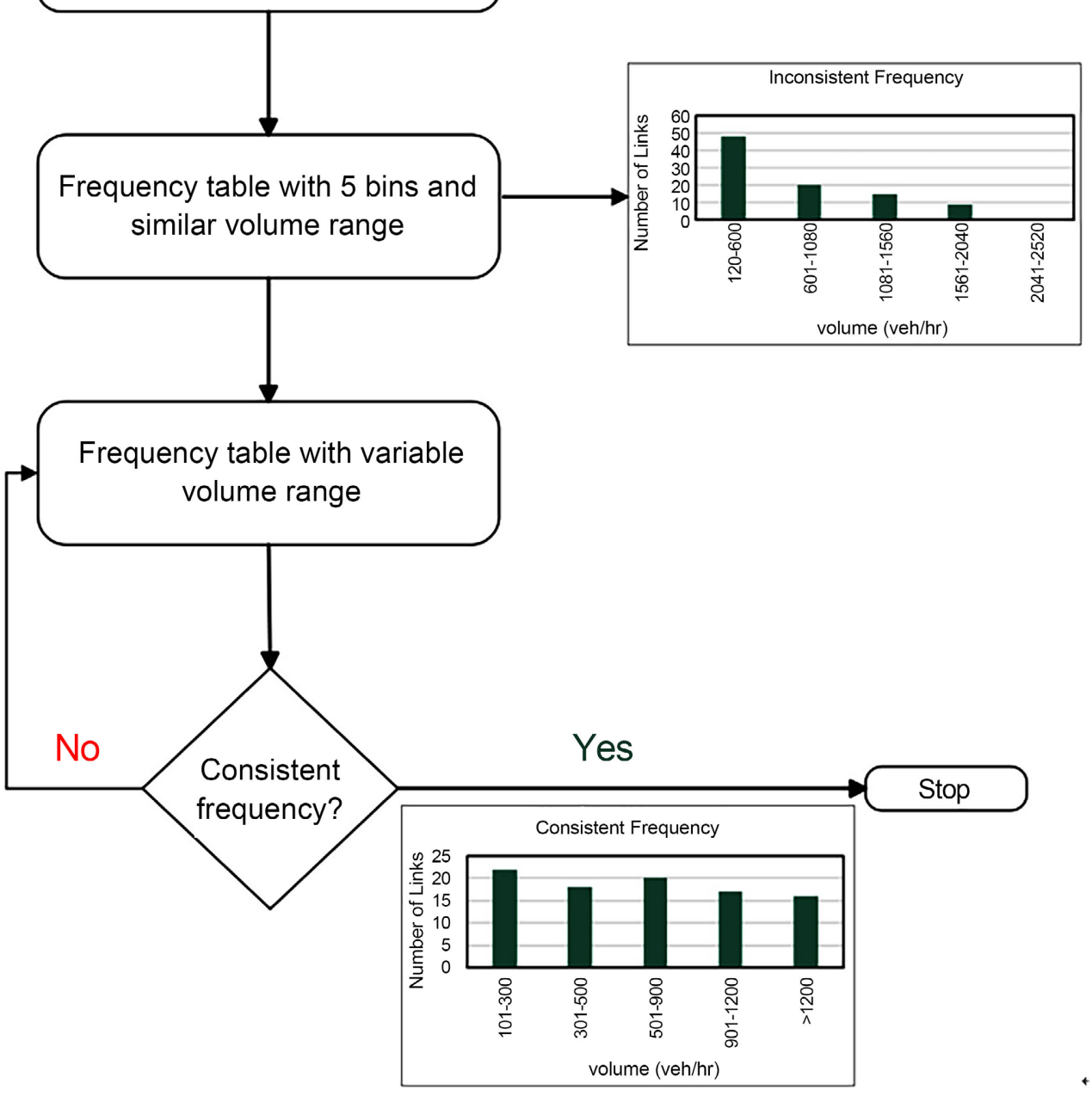

Figure 4. Group identification process. 


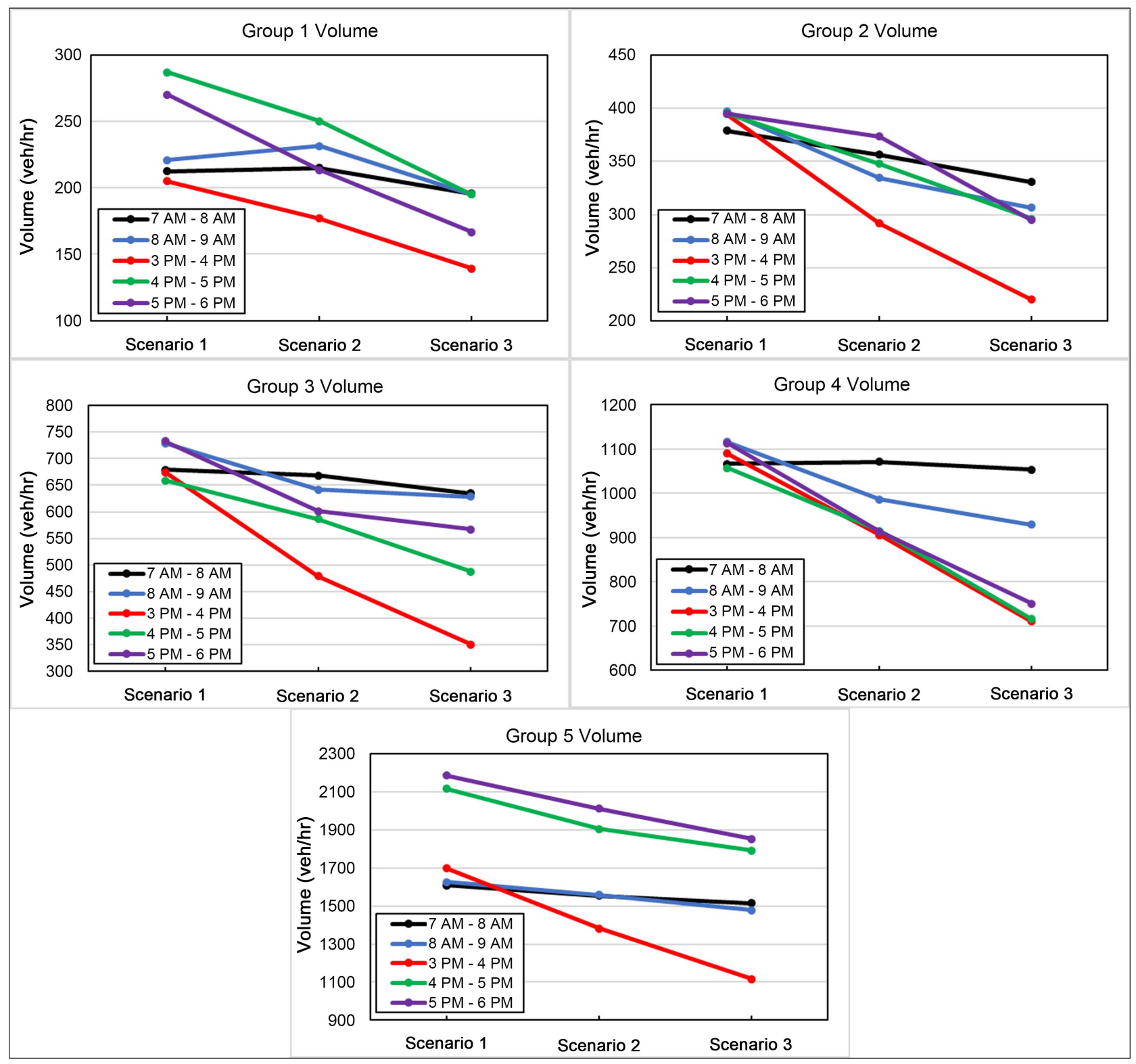

Figure 5. Traffic volume variation with increased transit ridership in 5 groups.

transit share of trips $=10.1 \%$ ). With increased transit ridership, a decrease in automobile trips is seen, especially in the PM time periods. It should be noted, that the effects on traffic volume between scenarios 1 and 2 are negligible under low traffic demand conditions during AM time periods (black and blue lines). It can be further observed that the mean traffic volume is reduced by $100 \mathrm{veh} / \mathrm{hr}$ from scenario 1 to scenario 3 from 4-6 PM (green and purple lines). A volume reduction (40 veh/hr) occurs also from scenario 2 to scenario 3 from 3-6 PM. Overall, the traffic volume for this group of roadway links is reduced due to the increase in public transit probability.

\subsubsection{Traffic Volume Change for Group 2}

Group 2 represents links with volume from 301 - 500 veh/hr. Group 2 shows 
traffic volume reduction throughout the 5 study time periods considered for increasing transit probability both from scenario 1 to scenario 2 and from scenario 1 to scenario 3 . The greatest impacts are observed during the $3 \mathrm{PM}$ to $4 \mathrm{PM}$ time period (red line) between scenarios 1 and 3 (nearly $170 \mathrm{veh} / \mathrm{hr}$ ), noticeable with the steep downward slope.

\subsubsection{Traffic Volume Change for Group 3}

From scenario 1 to scenario 3, all the time slots for group 3 (501 - $900 \mathrm{veh} / \mathrm{hr}$ ) show noticeable traffic volume reductions as the probability of transit use increases. The highest reduction ( $320 \mathrm{veh} / \mathrm{hr}$ ) takes place during the $3 \mathrm{PM}$ to $4 \mathrm{PM}$ time period (red line). The volume reduction is also noticeable from scenario 1 to scenario 2, from $3 \mathrm{PM}$ to $4 \mathrm{PM}$ (red line) and $5 \mathrm{PM}$ to $6 \mathrm{PM}$ (purple line) which show mean volume reductions of $300 \mathrm{veh} / \mathrm{hr}$ and $130 \mathrm{veh} / \mathrm{hr}$ respectively.

\subsubsection{Traffic Volume Change for Group 4}

Comparison of simulation outputs for Group 4 (901 - $1200 \mathrm{veh} / \mathrm{hr}$ ) links shows little to no change in the traffic volumes during the $7 \mathrm{AM}$ to $8 \mathrm{AM}$ time slot from scenario 1 to scenario 2 to scenario 3 (black line). During all other time periods, traffic volume drops as transit ridership increases. Once again, the highest such impact is observed during the $3 \mathrm{PM}$ to $4 \mathrm{PM}$ time slot (red line) where the mean traffic volume reduction between scenario 1 and 3 is $380 \mathrm{veh} / \mathrm{hr}$.

\subsubsection{Traffic Volume Change for Group 5}

In the group 5 links which carry volumes in excess of $1200 \mathrm{veh} / \mathrm{hr}$, mean traffic volumes are reduced for all 5 study timeframes and for both changes in transit probability considered (i.e., scenario 2 and 3 ) as compared to the scenario 1 . The most significant impact is observed during the $3 \mathrm{PM}$ to $4 \mathrm{PM}$ time period (red line) where mode shift toward transit (from scenario 1 to scenario 3) results in reduction of average traffic volume on group 5 links from $1700 \mathrm{veh} / \mathrm{hr}$ to 1100 $\mathrm{veh} / \mathrm{hr}$ (or $580 \mathrm{veh} / \mathrm{hr}$ ).

To further quantify the impacts on traffic volume as a result of changes in transit ridership, percentage flow reductions are calculated from no transit availability (base model) to scenarios 1, 2, and 3 that assume a future increase in transit ridership from $1.1 \%$ to $5.7 \%$ to $10.1 \%$ respectively. The results are summarized in Table 3 which are calculated using Equation (1), Equation (2), and Equation (3) below.

Table 3. Traffic volume reduction percentages in different scenarios

\begin{tabular}{lccc}
\hline Time Periods & $\begin{array}{c}\text { Base model to } \\
\text { scenario 1 }\end{array}$ & $\begin{array}{c}\text { Scenario 1 to } \\
\text { scenario 2 }\end{array}$ & $\begin{array}{c}\text { Scenario 1 to } \\
\text { scenario 3 }\end{array}$ \\
\hline 7 AM-8 AM & $-4.2 \%$ & $2.0 \%$ & $5.6 \%$ \\
8 AM-9 AM & $3.6 \%$ & $7.3 \%$ & $12.5 \%$ \\
3 PM-4 PM & $3.3 \%$ & $20.0 \%$ & $36.6 \%$ \\
4 PM-5 PM & $0.7 \%$ & $10.5 \%$ & $18.1 \%$ \\
5 PM-6 PM & $-3.4 \%$ & $9.1 \%$ & $16.8 \%$ \\
\hline
\end{tabular}




$$
\begin{aligned}
& \frac{\text { Base model volume }- \text { Scenario } 1 \text { volume }}{\text { Base model volume }} \times 100 \\
& \frac{\text { Scenario } 1 \text { volume }- \text { Scenario } 2 \text { volume }}{\text { Scenario } 1 \text { volume }} \times 100 \\
& \frac{\text { Scenario } 1 \text { volume }- \text { Scenario } 3 \text { volume }}{\text { Scenario } 1 \text { volume }} \times 100
\end{aligned}
$$

According to the results shown in Table 3, current transit ridership (scenario 1) had no impact in reducing traffic volume in three time periods (8 AM to 9 $\mathrm{AM}, 3 \mathrm{PM}$ to $4 \mathrm{PM}, 4 \mathrm{PM}$ to $5 \mathrm{PM}$ ), when compared to the no transit option (base). Increasing the transit ridership in scenario 2, resulted in noticeable traffic volume reductions. Further increase of the public transit ridership in scenario 3 reduces the traffic volumes even further, with the highest reduction percentage of $36.6 \%$ occurring between $3 \mathrm{PM}$ to $4 \mathrm{PM}$.

\subsection{Traffic Speed Analysis}

As the probability of choosing public transit is 0.1 in scenario 1 and then increased to 0.5 and 0.9 in scenarios 2 and 3 respectively, an improvement in traffic performance is expected in terms of speed increases associated with higher transit ridership. It is postulated that increased transit ridership will result in increases in the average vehicular speeds. Figure 6 shows the resulted speed for the three scenarios, 5 time slots and 5 groups studied.

\subsubsection{Traffic Speed Change for Group 1}

Free flow speed refers to the average speed which is traveled by a motorist in absence of congestion or adverse conditions in a roadway. The MATSim model results show that for free flow traffic conditions (group 1) the impact of mode shifts from automobile to transit on speed is negligible. As shown in Figure 6, speed differences are small in group 1 for all time periods considered when comparing results from scenario 1 to scenario 2 to scenario 3 .

\subsubsection{Traffic Speed Change for Group 2}

Group 2 represents near free flow conditions. For this traffic group, the effects on speed from ridership shifts toward transit are still small. As it can be observed from group 2 in Figure 6, speed increased overall by 2 to 3 mph except during the $3 \mathrm{PM}$ to $4 \mathrm{PM}$ (red line) time period.

\subsubsection{Traffic Speed Change for Group 3}

The study results confirm that for group 3 links, speeds increased by 3 to $4 \mathrm{mph}$ during the afternoon peak times (green line, $4 \mathrm{PM}$ to $5 \mathrm{PM}$ and purple line, 5 $\mathrm{PM}$ to $6 \mathrm{PM}$ ) both in scenario 2 and scenario 3. Speeds for the other three time periods remain almost constant or slightly decrease till the execution of scenario 3 for this group.

\subsubsection{Traffic Speed Change for Group 4}

Links in group 4 show no speed changes in response to shifts in ridership (red, 
blue and black lines. As transit ridership increases (from scenario 2 to 3 ) and during afternoon peak times (green line, $4 \mathrm{PM}$ to $5 \mathrm{PM}$ and purple line, $5 \mathrm{PM}$ to $6 \mathrm{PM}$ ) show overall speed increases of nearly $4 \mathrm{mph}$ and $7 \mathrm{mph}$ respectively.

\subsubsection{Traffic Speed Change for Group 5}

For study links with traffic conditions described by group 5, the highest speed increase happens during the afternoon peak (the purple line, $5 \mathrm{PM}$ to $6 \mathrm{PM}$ ) with the mean speed increase of $5 \mathrm{mph}$ in scenario 2 and $7 \mathrm{mph}$ in scenario 3 , as compared to scenario 1 . The second highest increase is visible from 4 PM to 5 PM (green line) with average speed increase of $3 \mathrm{mph}$ in scenario 2 and $5 \mathrm{mph}$ in scenario 3, as compared to scenario 1 . The speeds of remaining three time slots studied are almost similar throughout the three scenarios.

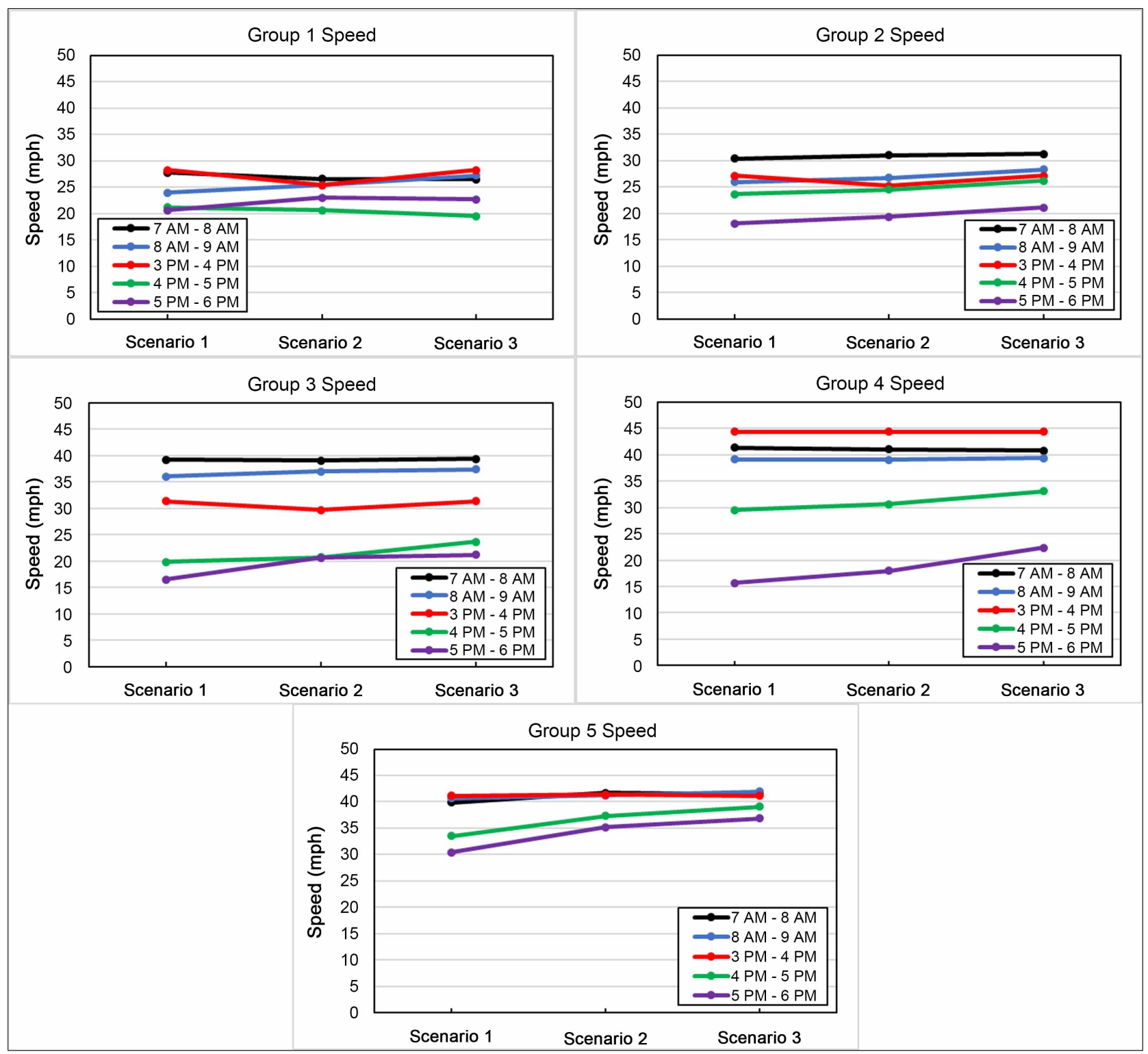

Figure 6. Traffic speed variation with increased transit ridership in 5 groups. 
Though the volume reduction is noticed to be higher for $3 \mathrm{PM}$ to $4 \mathrm{PM}$, speed increase for this time period is almost zero for group 1 to group 4 . Thus, free flow speeds of the 93 links were observed and compared with the operating speed for scenario 1 in $3 \mathrm{PM}$ to $4 \mathrm{PM}$. The findings from the observation showed that, most of the links, have near free flow condition (Table 4). Therefore, speed was not affected as a result of the volume reduction in scenario 2 and scenario 3.

A similar comparison setup was followed to document the percent speed increase resulting from the assumed increase in ridership in Birmingham as expressed by increased transit use probability in scenarios 2 and 3. The resulted percentage are summarized in Table 5 and are calculated using Equation (4), Equation (5), and Equation (6) below.

$$
\begin{aligned}
& \frac{\text { Scenario } 1 \text { speed }- \text { Base model speed }}{\text { Base model speed }} * 100 \\
& \frac{\text { Scenario } 2 \text { speed }- \text { Scenario } 1 \text { speed }}{\text { Scenario } 1 \text { speed }} * 100 \\
& \frac{\text { Scenario } 3 \text { speed }- \text { Scenario } 1 \text { speed }}{\text { Scenario } 1 \text { speed }} * 100
\end{aligned}
$$

Table 4. Traffic speed changes for different scenarios.

\begin{tabular}{cccc}
\hline Link ID & $\begin{array}{c}\text { Operating speed in } \\
\text { scenario } 1 \text { (mph) }\end{array}$ & $\begin{array}{c}\text { Free Flow } \\
\text { speed (mph) }\end{array}$ & $\begin{array}{c}\text { Difference between Free flow } \\
\text { and operating speed (mph) }\end{array}$ \\
\hline 107920507_14 & 36 & 37 & 1 \\
259336961_0 & 36 & 37 & 1 \\
323899401_8 & 49 & 50 & 1 \\
7782325_7_r & 37 & 37 & 0 \\
259970324_1_r & 26 & 28 & 2 \\
7742120_1_r & 36 & 37 & 1 \\
592215806_4_r & 37 & 37 & 0 \\
259311994_2 & 36 & 37 & 1 \\
7740932_2 & 27 & 28 & 1 \\
394283610_3 & 19 & 19 & 0 \\
7782325_5 & 37 & 37 & 0 \\
$7740932 \_0$ & 28 & 28 & 0 \\
\hline
\end{tabular}

Table 5. Traffic speed changes for different scenarios.

\begin{tabular}{lccc}
\hline Time Periods & $\begin{array}{c}\text { Base model to } \\
\text { scenario 1 }\end{array}$ & $\begin{array}{c}\text { Scenario 1 to } \\
\text { scenario 2 }\end{array}$ & $\begin{array}{c}\text { Scenario 1 to } \\
\text { scenario 3 }\end{array}$ \\
\hline 7 AM-8 AM & $0.4 \%$ & $0.2 \%$ & $0.2 \%$ \\
8 AM-9 AM & $0.1 \%$ & $2.1 \%$ & $4.8 \%$ \\
3 PM-4 PM & $8.8 \%$ & $-2.5 \%$ & $0.0 \%$ \\
4 PM-5 PM & $-2.4 \%$ & $8.8 \%$ & $15.2 \%$ \\
5 PM-6 PM & $-2.3 \%$ & $16.0 \%$ & $22.0 \%$ \\
\hline
\end{tabular}


According to the results shown in Table 5, the increase in transit ridership contributes to increasing the speed during peak traffic time periods. The largest increases in speeds were found between $4 \mathrm{PM}$ to $5 \mathrm{PM}$ and $5 \mathrm{PM}$ to $6 \mathrm{PM}$ while speeds in remaining three time periods had very little or no increase in speed.

\subsection{Travel Time Analysis}

Travel time for a roadway link refers to the time needed to drive from start point to the end point of that link. The expectation is that modal shifts from automobile to transit may result in reduction of link travel times, thus resulting in an improvement of traffic network performance. To verify this expectation, an analysis of travel time data was performed for the study links and for the 5 study time periods. Average travel times for the three scenarios and 5 groups are shown in Figure 7.

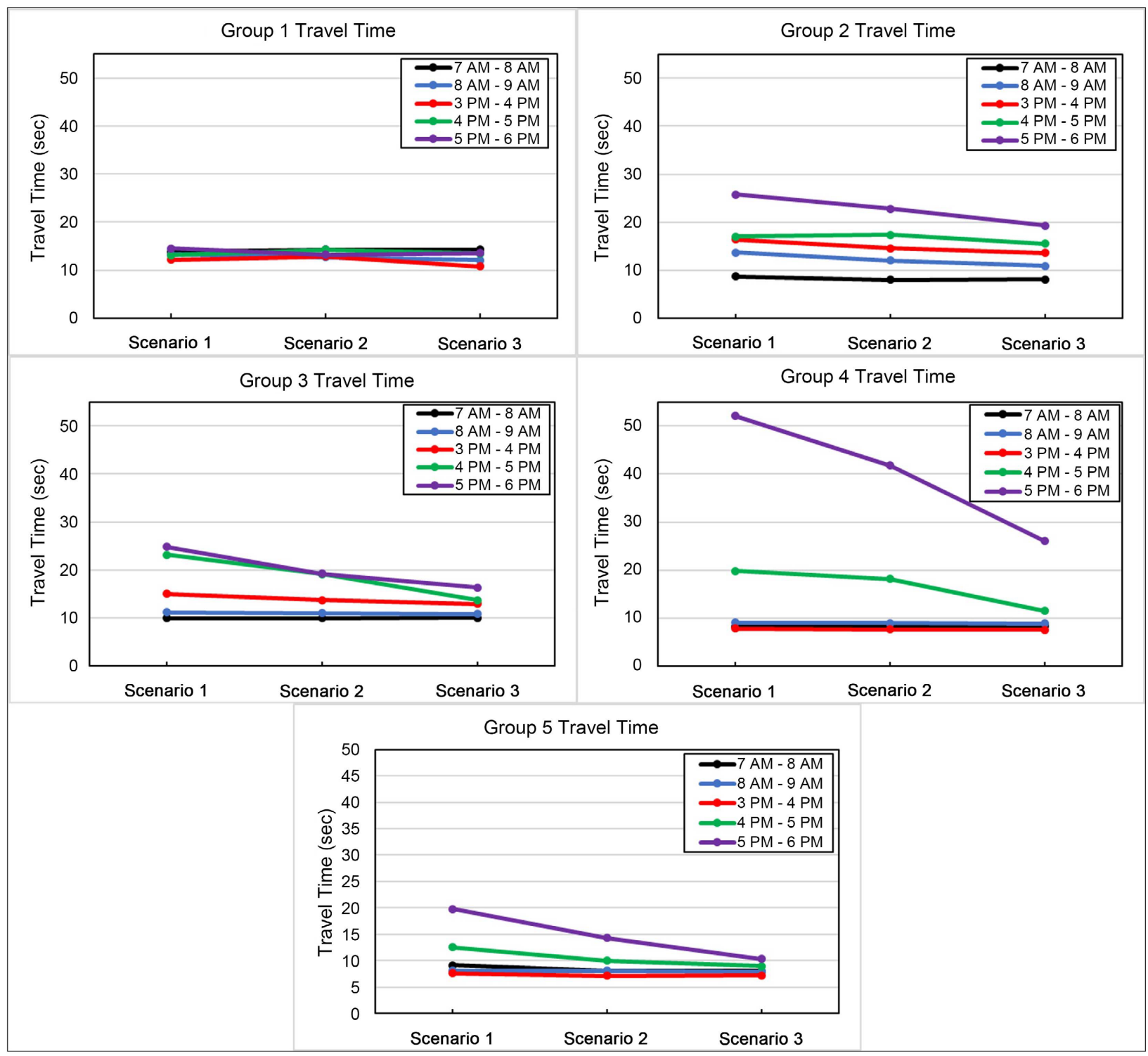

Figure 7. Travel Time variation with increased transit ridership in 5 groups. 


\subsubsection{Travel Time Change for Group 1}

The impacts on average travel times in response to modal shifts towards transit are small ( 1 to $2 \mathrm{sec}$ ) for all time slots considered in group 1 .

\subsubsection{Travel Time Change for Group 2}

Under group 2 conditions, there is little to no change observed to average travel time as transit ridership increases from scenario 1 to scenario 2 and from scenario 1 to scenario 3 . An average travel time decrease by around 3 seconds 5 PM to $6 \mathrm{PM}$ (purple line) from scenario 1 to 2 and around 6.5 seconds from scenario 1 to scenario 3 , represents the highest decrease of this group.

\subsubsection{Travel Time Change for Group 3}

Under group 3 traffic conditions, some reductions in travel times are realized during the afternoon peak time periods (green line, $4 \mathrm{PM}$ to $5 \mathrm{PM}$ and purple line, $5 \mathrm{PM}$ to $6 \mathrm{PM}$ ) as transit ridership increases in scenario 2 and scenario 3. The average travel time is decreased by almost 10 seconds from 4 PM to 5 PM in scenario 3 and almost 8 seconds 5 PM to 6 PM.

\subsubsection{Travel Time Change for Group 4}

There is no visible impact on link travel times from changes in transit ridership during the $7 \mathrm{AM}$ to $8 \mathrm{AM}$ (black line), $8 \mathrm{AM}$ to $9 \mathrm{AM}$ (blue line) and $3 \mathrm{PM}$ to 4 PM (red line) time periods in group 4. The opposite is true for the afternoon peak times, as shown by the green line and purple line in Figure 7.

Based on the simulation results, the average travel time during the 5 PM to 6 PM time period (purple line) decreased by 9 seconds from scenario 1 to scenario 2 and a total of 26 seconds from scenario 1 to scenario 3 . The decrease in travel times during the $4 \mathrm{PM}$ to $5 \mathrm{PM}$ time period (green line) are around 1 second and 8 seconds between scenario 1 and scenarios 2 and 3 respectively.

\subsubsection{Travel Time Change for Group 5}

From the simulation results for group 5 conditions, it can be seen that during 7 $\mathrm{AM}$ to $8 \mathrm{AM}, 8 \mathrm{AM}$ to $9 \mathrm{AM}$ and $3 \mathrm{PM}$ to $4 \mathrm{PM}$ (black, blue and red lines respectively) there is very little or no change in average link travel times for the two scenarios considered as compared with scenario 1 . However, during the 4 $\mathrm{PM}$ to $5 \mathrm{PM}$ time period (green line) as well as the $5 \mathrm{PM}$ to $6 \mathrm{PM}$ time period (purple line), link travel times decreased by 3 seconds and 9 seconds respectively, when transit ridership changed from scenario 1 to scenario 3 conditions. Using the same comparison setup as the one used for determining impacts on traffic volumes and speed, the percent change of average travel time is calculated using Equation (7), Equation (8) and Equation (9). The results are reported in Table 6.

$$
\begin{aligned}
& \frac{\text { Base model travel time }- \text { Scenario1 travel time }}{\text { Base model travel time }} * 100 \\
& \frac{\text { Scenario } 1 \text { travel time }- \text { Scenario } 2 \text { travel time }}{\text { Scenario } 1 \text { travel time }} * 100
\end{aligned}
$$


Table 6. Travel Time percentage change in different scenarios.

\begin{tabular}{cccc}
\hline Time Periods & $\begin{array}{c}\text { Base model to } \\
\text { scenario 1 }\end{array}$ & $\begin{array}{c}\text { Scenario 1 to } \\
\text { scenario 2 }\end{array}$ & $\begin{array}{c}\text { Scenario 1 to } \\
\text { scenario 3 }\end{array}$ \\
\hline 7 AM-8 AM & $0.8 \%$ & $2.3 \%$ & $1.9 \%$ \\
8 AM-9 AM & $-1.0 \%$ & $4.5 \%$ & $8.5 \%$ \\
3 PM-4 PM & $10.3 \%$ & $6.8 \%$ & $11.4 \%$ \\
4 PM-5 PM & $-2.4 \%$ & $13.8 \%$ & $29.1 \%$ \\
5 PM-6 PM & $-5.2 \%$ & $23.1 \%$ & $40.7 \%$ \\
\hline
\end{tabular}

$$
\frac{\text { Scenario } 1 \text { travel time }- \text { Scenario } 3 \text { travel time }}{\text { Scenario } 1 \text { travel time }} * 100
$$

The results indicate that transit ridership during study time has no effect in reducing average travel time (except for the time between 3 PM to $4 \mathrm{PM}$ ), however, travel time is reduced while transit ridership is increased in scenario 2 and scenario 3 . The reduction is higher for the scenario with more transit probability (i.e., scenario 3 ) and for groups 4 and 5 where the network carries heavier traffic loads.

\subsection{Significance Test Analysis}

To understand whether the traffic flow reduction associated with changes in transit ridership is statistically significant, several significance tests were performed. First, a significance test was performed to test difference within the three scenarios. If the traffic flow reduction was significant within the scenarios, then tests between the scenarios were also performed. Table 7 shows the p-value of the significance tests performed herein. The tests were performed using the $95 \%$ confidence level, hence, a p-value less than 0.05 indicates that the difference is statistically significant between/within groups.

The results from the statistical tests between the base model and scenario 1 imply that the current level of public transit use does not have any significant impact on traffic volumes. This is evident from the high p-values documented in Table 7 resulting from the comparison of traffic volumes between the base model and with scenario 1. Furthermore, results show that traffic flow reduction is statistically significant when comparing results from scenario 1 to scenario 2 as well as scenario 1 to scenario 3 . The only exception is for group 1 which shows a p-value of $0.224>0.05$, indicating that there is no evidence to support that there is a statistical difference in traffic volumes from scenario 1 to scenario 2 during free flow conditions (group 1).

Table 8 and Table 9 show the p-values of the significance tests done for speed and travel time changes respectively. The results show that there is not enough evidence to suggest that there is statistically significant differences in speed or travel time associated with the increase in transit ridership as per the study scenarios. 
Table 7. Statistical significance test scores (p-value) for traffic volume changes.

\begin{tabular}{lcccc}
\hline Groups & $\begin{array}{c}\text { Base model with } \\
\text { scenario 1 }\end{array}$ & $\begin{array}{c}\text { Within three } \\
\text { scenarios }\end{array}$ & $\begin{array}{c}\text { Scenario 1 with } \\
\text { scenario 2 }\end{array}$ & $\begin{array}{c}\text { Scenario 1 with } \\
\text { scenario 3 }\end{array}$ \\
\hline Group 1 & 0.7801 & $\mathbf{0 . 0 1 6 9}$ & 0.2240 & $\mathbf{0 . 0 1 7 5}$ \\
Group 2 & 0.6078 & $\mathbf{0 . 0 0 0 6}$ & $\mathbf{0 . 0 2 2 2}$ & $\mathbf{0 . 0 0 5 5}$ \\
Group 3 & 0.5706 & $\mathbf{0 . 0 2 8 5}$ & $\mathbf{0 . 0 3 2 3}$ & $\mathbf{0 . 0 3 2 7}$ \\
Group 4 & 0.6728 & $\mathbf{0 . 0 0 5 0}$ & $\mathbf{0 . 0 1 2}$ & $\mathbf{0 . 0 2 1}$ \\
Group 5 & 0.9968 & 0.2827 & & \\
\hline
\end{tabular}

Table 8. Statistical significance test scores (p-values) for speed changes.

\begin{tabular}{ccc}
\hline Groups & Base model with scenario 1 & Within three scenarios \\
\hline Group 1 & 0.8958 & 0.9517 \\
Group 2 & 0.6599 & 0.7837 \\
Group 3 & 0.8967 & 0.9394 \\
Group 4 & 0.9413 & 0.9536 \\
Group 5 & 0.9322 & 0.2999 \\
\hline
\end{tabular}

Table 9. Statistical significance test scores (p-values) for travel time changes.

\begin{tabular}{ccc}
\hline Groups & Base model with scenario 1 & Within three scenarios \\
\hline Group 1 & 0.9738 & 0.5976 \\
Group 2 & 0.7644 & 0.7221 \\
Group 3 & 0.7142 & 0.4394 \\
Group 4 & 0.8730 & 0.7504 \\
Group 5 & 0.6853 & 0.4117 \\
\hline
\end{tabular}

\section{Conclusions and Recommendations}

The study used a comprehensive activity-based simulation model of the Birmingham area to simulate traffic operations under various transit ridership scenarios ranging from $0 \%$ (base) to $1.1 \%$ (scenario 1 -current) to $5.7 \%$ (scenario 2 -future) to $10.1 \%$ (scenario 3-future). The analysis considered inks with various levels of traffic demand (Groups 1 to 5 ) and 5 hourly time slots. The main findings from this study are summarized below.

1) Current transit ridership has no significant effect on traffic volume reduction for the roadway sections with low traffic demand. As transit ridership increases, traffic volume reductions are reported and, with a few exceptions, the traffic flow reductions are statistically significant when comparing results from scenario 1 to scenario 2 as well as scenario 1 to scenario 3 .

2) Increase the transit ridership results in speed increases for 4 of the 5 time periods considered in this study, however, those changes are not statistically significant. 
3) Similarly, with the increase of transit ridership, travel time is decreasing, but not significantly.

Based on the study findings it is concluded that higher levels of modal shifts from private cars to transit modes might be necessary in order to materialize significant differences in speed and travel time. Still, some network performance improvement as a result of increased transit ridership was documented in this study as demonstrated by the percent reduction of volume and travel times, and percent increase of speed. This implies that the benefits of increasing transit ridership in medium sized cities like Birmingham can contribute to improving the performance of the road network.

Initiatives that can increase transit ridership in the given road network include expanding the number of bus routes and/or frequency of service, strategically positioning new bus stops near the residential areas, providing transit use incentives, and improving the quality of transit service. Additionally, initiating a feeder service to provide first and last mile service connections for distant passengers shows a good potential to increase transit ridership.

The findings from this study highlight the potential benefits of increased bus ridership on transportation network operations in medium sized US cities such as Birmingham. The study results may encourage the local transit authority, transportation planners and decision makers to think of ways to improve and/or expand public transit services in order to attract new public transit users. The investment for those improvements can be justified using the potential transportation network operational performance benefits deduced by this study.

\section{Acknowledgements}

The study was conducted under the US DOT-STRIDE sponsored project (STRIDE I2) "Mitigating Network Congestion by Integrating Transportation Network Companies and Urban Transit".

\section{Conflicts of Interest}

The authors declare no conflicts of interest regarding the publication of this paper.

\section{References}

[1] Litman, T. (2020) Evaluating Public Transit Benefits and Costs. Victoria Transport Policy Institute, Victoria.

[2] Pachauri, R.K., et al. (2014) AR5 Synthesis Report: Climate Change 2014. Contribution of Working Groups I, II and III to the Fifth Assessment Report of the Intergovernmental Panel on Climate Change. Intergovernmental Panel on Climate Change, Geneva.

[3] Levy, J.I., Buonocore, J.J. and Von Stackelberg, K. (2010) Evaluation of the Public Health Impacts of Traffic Congestion: A Health Risk Assessment. Environmental Health, 9, Article No. 65. https://doi.org/10.1186/1476-069X-9-65

[4] Blumenberg, E., Garrett, M., King, H., Paul, J., Ruvolo, M., Schouten, A., et al. (2020) 
What's Behind Recent Transit Ridership Trends in the Bay Area? Volume I: Overview and Analysis of Underlying Factors. UCLA Institute of Transportation Studies, Los Angeles. https://doi.org/10.17610/T6PC7Q

[5] Jensen, M. (1999) Passion and Heart in Transport-A Sociological Analysis on Transport Behaviour. Transport Policy, 6, 19-33. https://doi.org/10.1016/S0967-070X(98)00029-8

[6] Cools, M., Moons, E., Janssens, B. and Wets, G. (2009) Shifting towards Environment-Friendly Modes: Profiling Travelers Using Q-Methodology. Transportation, 36, 437-453. https://doi.org/10.1007/s11116-009-9206-Z

[7] Gärling, T., Gillholm, R. and Gärling, A. (1998) Reintroducing Attitude Theory in Travel Behavior Research: The Validity of an Interactive Interview Procedure to Predict Car Use. Transportation, 25, 129-146. https://doi.org/10.1023/A:1005004311776

[8] Tao, S., He, S.Y. and Thøgersen, J. (2019) The Role of Car Ownership in Attitudes towards Public Transport: A Comparative Study of Guangzhou and Brisbane. Transportation Research Part F: Traffic Psychology and Behaviour, 60, 685-699. https://doi.org/10.1016/j.trf.2018.12.005

[9] Kwan, S.C. and Hashim, J.H. (2016) A Review on Co-Benefits of Mass Public Transportation in Climate Change Mitigation. Sustainable Cities and Society, 22, 11-18. https://doi.org/10.1016/j.scs.2016.01.004

[10] Beaudoin, J., Farzin, Y.H. and Lawell, C.-Y.C.L. (2015) Public Transit Investment and Sustainable Transportation: A Review of Studies of Transit's Impact on Traffic Congestion and Air Quality. Research in Transportation Economics, 52, 15-22. https://doi.org/10.1016/j.retrec.2015.10.004

[11] Shapiro, R.J., Hassett, K.A. and Arnold, F.S. (2016) Conserving Energy and Preserving the Environment: The Role of Public Transportation. Report, American Public Transportation Association, Washington DC.

[12] Weisbrod, G. and Reno, A. (2009) Economic Impact of Public Transportation Investment. Citeseer.

[13] Litman, T. (2009) Transportation Cost and Benefit Analysis. Vol. 31, Victoria Transport Policy Institute, Victoria.

[14] CDC (Centers for Disease Control and Prevention) (2005) Adult Participation in Recommended Levels of Physical Activity-United States, 2001 and 2003. Morbidity and Mortality Weekly Report, 54, 1208-1212.

https://www.cdc.gov/mmwr/preview/mmwrhtml/mm5447a3.htm\#: :text=The\%20f indings\%20in\%20this\%20report,and\%2045.9\%25\%2C\%20respectively

[15] Lachapelle, U. and Frank, L.D. (2009) Transit and Health: Mode of Transport, Employer-Sponsored Public Transit Pass Programs, and Physical Activity. Journal of Public Health Policy, 30, S73-S94. https://doi.org/10.1057/jphp.2008.52

[16] Litman, T. (2015) Evaluating Public Transportation Health Benefits. Victoria Transport Policy Institute for the American Public Transportation Association, Victoria, BC, Canada.

[17] Freeland, A.L., Banerjee, S.N., Dannenberg, A.L. and Wendel, A.M. (2013) Walking Associated with Public Transit: Moving toward Increased Physical Activity in the United States. American Journal of Public Health, 103, 536-542. https://doi.org/10.2105/AJPH.2012.300912

[18] Jou, R.-C. and Chen, T.-Y. (2014) Factors Affecting Public Transportation, Car, and Motorcycle Usage. Transportation Research Part A: Policy and Practice, 61, 186-198. https://doi.org/10.1016/j.tra.2014.02.011 
[19] Bay Area Rapid Transit (BART) (2016) BART's Role in the Region. Bay Area Rapid Transit, Oakland.

[20] Adler, M.W. and van Ommeren, J.N. (2016) Does Public Transit Reduce Car Travel Externalities? Quasi-Natural Experiments' Evidence from Transit Strikes. Journal of Urban Economics, 92, 106-119. https://doi.org/10.1016/j.jue.2016.01.001

[21] Anderson, M.L. (2014) Subways, Strikes, and Slowdowns: The Impacts of Public Transit on Traffic Congestion. American Economic Review, 104, 2763-2796. https://doi.org/10.1257/aer.104.9.2763

[22] Ewing, R., Tian, G. and Spain, A. (2014) Effect of Light-Rail Transit on Traffic in a Travel Corridor. Transportation Research and Education Center, Portland. https://doi.org/10.15760/trec.56

[23] Liu, Y. and Cirillo, C. (2015) Measuring Transit Service Impacts on Vehicle Ownership and Use. Public Transport, 7, 203-222. https://doi.org/10.1007/s12469-014-0098-8

[24] Mulalic, I., Pilegaard, N. and Rouwendal, J. (2016) Does Improving Public Transport Decrease Car Ownership? Evidence from the Copenhagen Metropolitan Area. Discussion Paper 15-139/VIII, Tinbergen Institute, Amsterdam. https://doi.org/10.2139/ssrn.2710547

[25] Sisiopiku, V.P. and Salman, F. (2019) Simulation Options for Modeling Shared Mobility. Proceedings of the 2019 AlaSim International Conference and Exhibition, Huntsville, AL, 24 October 2019.

[26] Sisiopiku, V.P., Hadi, M., McDonald, N., Steiner, R. and Ramadan, O.E. (2019). Technology Influence on Travel Demand and Behaviors (Project B). Final Report to the Southeastern Transportation Research, Innovation, Development and Education Center (STRIDE), December 2019.

[27] Horni, A., Nagel, K. and Axhausen, K.W., Eds. (2016) The Multi-Agent Transport Simulation MATSim. Ubiquity Press, London.

[28] Guo, G., Khalil, J.M., Yan, D. and Sisiopiku, V. (2019) Realistic Transport Simulation: Tackling the Small Data Challenge with Open Data. 2019 IEEE International Conference on Big Data (Big Data), Los Angeles, 9-12 December 2019, 4512-4519. https://doi.org/10.1109/BigData47090.2019.9006457

[29] Bischoff, J., Márquez-Fernández, F.J., Domingues-Olavarría, G., Maciejewski, M. and Nagel, K. (2019) Impacts of Vehicle Fleet Electrification in Sweden-A SimulationBased Assessment of Long-Distance Trips. 2019 6th International Conference on Models and Technologies for Intelligent Transportation Systems (MT-ITS), Cracow, 5-7 June 2019, 1-7. https://doi.org/10.1109/MTITS.2019.8883384

[30] MATSim (n.d.) Scenario Gallery. https://matsim.org/gallery/berlin

[31] BJTCA (Birmingham-Jefferson County Transit Authority) (2018) Transit Development Plan-Technical Memorandum 1. Birmingham-Jefferson County Transit Authority, Birmingham.

[32] U.S. Census Bureau (2019) American Community Survey 1-Year Estimates. https://censusreporter.org/profiles/31000US13820-birmingham-hoover-al-metro-area/

[33] Van der Merwe, J. (2011) Agent-Based Transport Demand Modelling for the South African Commuter Environment. University of Pretoria, Pretoria.

[34] TomTom (2020) Birmingham Traffic. https://www.tomtom.com/en_gb/traffic-index/birmingham-alabama-traffic/ 